\title{
Estudio de validación de cuatro diferentes criterios para el diagnóstico de sindrome metabólico en población infantil
}

\author{
Validation study of four different criteria for the \\ diagnosis of metabolic syndrome in children
}

\author{
Yesika-N Valdés-Villalpando; Julio-C Campuzano; ${ }^{1}$ Luisa-María Sánchez-Zamorano ${ }^{1}$; Victor Bermudez-Morales ${ }^{1}$; \\ Oscar Peralta-Zaragoza ${ }^{1}$; Cinthya Díaz-Benítez ${ }^{1}$; Miguel Cruz²; Ana-I Burguete-García ${ }^{1}$
}

Forma de citar: Valdés Villapardo Y, Campuzano J, Sánchez Zamorano LM, Bermudez Morales V, Peralta Zaragoza O, Díaz Benítez C, et al. Estudio de validación de cuatro diferentes criterios para el diagnóstico de síndrome metabólico en población infantil. Rev Univ Ind Santander Salud. 50(2): 126-135. doi: http://dx.doi.org/10.18273/revsal.v50n2-2018004 @) (1)

\section{Resumen}

Introducción: El síndrome metabólico (SM) es un problema de salud pública, el cual no cuenta con estrategias adecuadas de prevención, diagnóstico y tratamiento para población infantil. Los criterios existentes son controversiales y no son aplicables en los niños. Asimismo, varían según autores y comités de expertos; lo que podría tener importantes consecuencias en el diagnóstico de SM, impactando el tratamiento oportuno y el pronóstico del individuo. Objetivo: Validar criterios (NCEP-ATPIII; Cook, Ford y Duncan, et al; Ferranti, et al; Cruz, et al; e $\mathrm{IDF}^{1}$ ) para el diagnóstico de SM en niños mexicanos. Metodología: Estudio transversal de 2599 niños entre 6 y 16 años, residentes de la Ciudad de México. Se consideró SM con tres o más de los cinco componentes en los distintos criterios; y dos o más componentes con la presencia de obesidad central para IDF. Se consideró como Gold Standard la combinación de los cinco criterios diagnósticos. Para identificar el mejor valor predictivo se calculó sensibilidad, especificidad, valor predictivo positivo (VPP), valor predictivo negativo (VPN) y razón de verosimilitud. Resultados: Se observó una mayor proporción de individuos diagnosticados con SM con el criterio de Ferranti, et al. en comparación con los demás criterios evaluados. Nuestra propuesta ad hoc presentó una alta sensibilidad $(0,89)$ y especificidad $(0,90)$ frente al Gold Standard aplicado. Conclusión: El criterio propuesto por nosotros contiene una elección de componentes sencillos y de bajo costo, que facilitará su aplicación, permitiendo la unificación en el diagnóstico, tratamiento y pronóstico poblacional, reduciendo los índices de morbimortalidad en mexicanos.

Palabras clave: Diagnóstico; Síndrome X metabólico; Niños.

\begin{abstract}
Introduction Metabolic syndrome (MS) is a public health problem without appropriate strategies for prevention, diagnosis and treatment in children. Existing criteria are controversial and not applicable for pediatric population, with variations according to different authors and expert committees, which could have important consequences

1. Instituto Nacional de Salud Pública. Cuernavaca, México.

2. Centro Médico Nacional Siglo XXI. Ciudad de México, México.

Correspondencia: Ana Isabel Burguete García. Dirección: Instituto Nacional de Salud Pública, Av. Universidad 655, Col. Santa María Ahuacatitlán, Cuernavaca. Teléfono: \pm 5217773293000 Ext: 2886. Correo electrónico: aburguete@insp.mx
\end{abstract}


in MS diagnosis, treatment and prognosis. Objective: To validate different definitions (NCEP-ATPIII; Cook, Ford and Duncan, et al; Ferranti, et al; Cruz, et al; and $\mathrm{IDF}^{1}$ ) for metabolic syndrome diagnosis in Mexican children. Methodology: Cross-sectional study of 2599 children aged between 6 and 16 years, residents of Mexico City. MS was defined as the presence of three or more of the five components in the different criteria; and two or more components with the presence of central obesity for IDF. The Gold Standard was considered as the combination of the five diagnostic criteria. To identify the best predictive value, sensitivity, specificity, positive predictive value (PPV), negative predictive value (NPV) and likelihood ratio were calculated. Results: A greater proportion of individuals diagnosed with the Ferranti, et al criterion was observed in comparison with the other criteria evaluated. We proposed an ad hoc criteria which showed a high sensitivity $(0,89)$ and specificity $(0,90)$ compared to the Gold Standard applied. Conclusion: Our diagnostic criteria contains a choice of simple and low-cost components that will facilitate its application in health institutions and will unify= diagnostic criteria, treatment, and prognosis, reducing morbidity and mortality rates in Mexican population.

Keywords: Diagnosis; Metabolic syndrome X; Children.

\section{Introducción}

La presencia de síndrome metabólico (SM) en niños obesos es un problema actual de salud pública, hecho que ha incrementado la preocupación por incorporar prácticas preventivas, promocionando el diagnóstico temprano de obesidad y evaluando sus condiciones de comorbilidad $^{1-4}$. No obstante, aún no existen estrategias adecuadas de prevención, diagnóstico y tratamiento eficaces para la mayoría de los casos. Por esta razón, la obesidad y el SM se han convertido en un serio problema de salud pública en nuestro país ${ }^{4}$; reportándose múltiples causas como origen del problema en el diagnóstico y tratamiento oportuno de estos padecimientos ${ }^{3-5}$ :

Por otro lado, existe controversia con relación al diagnóstico de SM en adultos, en donde se emplean varios criterios, dentro de los que se encuentran, el Programa Nacional de Educación sobre el Colesterol y el Panel III de Tratamiento del Adulto (NCEP- ATP III) $)^{1,6}$, la Organización Mundial de la Salud (OMS), la Federación Internacional de Diabetes (IDF) ${ }^{1,6}$ y el Grupo Europeo de Estudio de Resistencia a la Insulina (EGIR), entre otros ${ }^{6}$. En niños, los criterios diagnósticos de SM son aún más controversiales debido a que los valores considerados en el adulto no son aplicables en este grupo etáreo, debe considerarse la dinámica del crecimiento y desarrollo (especialmente durante los períodos de rápido crecimiento) en donde la sensibilidad a la insulina, la presión arterial, los niveles de lípidos, y la distribución de las dimensiones del organismo cambian con la edad $d^{1,3,5,7,8}$.

Los criterios diagnósticos de SM varían según los autores y los comités de expertos. Las diferencias entre ellos se centran en el peso que asume cada componente o factor de riesgo considerado para el diagnóstico de $\mathrm{SM}$, así como en los puntos de corte establecidos en 127 cada una de las definiciones; esto ha ocasionado que, a la propia variabilidad de la prevalencia del SM en diferentes poblaciones, se agreguen las diferencias derivadas del empleo de varios criterios para definirlo, ocasionado dificultades al comparar poblaciones ${ }^{5,7,9,10}$. Estas diferencias son relevantes y tienen importantes consecuencias debido a que según el criterio diagnóstico aplicado, un individuo será diagnosticado o no de SM, la prevalencia reportada variará, y lo que es más importante, también lo harán el tratamiento y el pronóstico del individuo ${ }^{11}$. Considerando lo anterior, el presente estudio tuvo como objetivo validar diferentes definiciones (NCEP-ATPIII; IDF; Cook, Ford y Duncan, et al; Ferranti, et al; Cruz $)^{1,6,12-15}$. para el diagnóstico de síndrome metabólico en niños mexicanos ${ }^{1}$.

\section{Metodología}

Población y diseño de estudio: Estudio transversal realizado en 2599 niños entre 6 y 16 años, no relacionados, residentes de la Ciudad de México (Zona Poniente, Oriente, Norte y Sur). Se seleccionaron aquellos niños sin diagnóstico de diabetes, hipertensión arterial y enfermedades cardiovasculares, a los cuales se les pidió su asentimiento para la participación en el estudio, así como la firma del consentimiento informado a sus padres o tutores. Se descartaron aquellos niños que, al momento de la entrevista, hubieran tomado antibióticos en los dos meses previos o presentaran enfermedades infecciosas.

Personal previamente capacitado recopiló información mediante un cuestionario estructurado, sobre información socioeconómica, demográfica, heredofamiliar, de hábitos dietéticos y actividad física; además de realizar la toma de muestras biológicas y medidas antropométricas. 


\section{Variables de interés utilizadas para el DX de SM}

Medidas Antropométricas: Personal previamente estandarizado y capacitado realizó las medidas antropométricas. El peso $(\mathrm{kg})$ se obtuvo a través de la báscula SECA clara, modelo 803 con precisión de $0,1 \mathrm{Kg}$ (SECA, Hamburg, Germany), la talla (cm) se obtuvo con un estadiómetro portátil con precisión de $0,1 \mathrm{~cm}$, modelo 213 (SECA, Hamburg, Germany), la circunferencia de cintura $(\mathrm{cm})$ y circunferencia de cadera $(\mathrm{cm})$ se realizaron con la cinta métrica flexible de fibra de vidrio SECA, modelo 201, con precisión de hasta 1 milímetro y alcance de $205 \mathrm{~cm}$. Se calculó el IMC $\left(\mathrm{Kg} / \mathrm{m}^{2}\right)$ utilizando las referencias de crecimiento para niños de 5 a 19 años de la OMS. El Índice Cintura Cadera (ICC) fue calculado dividiendo la circunferencia de cintura $(\mathrm{cm})$ entre la circunferencia de cadera $(\mathrm{cm})$.

Mediciones Bioquímicas: Posterior a un ayuno de 12 horas, a los participantes del estudio se les tomó una muestra de sangre venosa de $5 \mathrm{ml}$, de la cual se extrajo el suero y se conservó a $-20^{\circ} \mathrm{C}$ hasta el momento del análisis. Las muestras séricas fueron procesadas antes de las 24 horas de la toma, en el Equipo Clinical Chemistry System ILAB 300 plus ${ }^{\circledR}$ para GA, CT, C-LDL, C-HDL y TGC y la determinación de Insulina se realizó por quimioluminiscencia (INMULITE ${ }^{\circledR}$ ). Se utilizaron controles de calidad y curvas de calibración para todas las mediciones siguiendo el protocolo de los fabricantes. Para obtener el coeficiente de variación (CV) inter-ensayo e intra-ensayo, las mediciones fueron realizadas en duplicado y se utilizó una mezcla de sueros de distintos niños del proyecto en todos los ensayos; obteniendo un $\mathrm{CV}$ intra-ensayo $<8 \%$ y un $\mathrm{CV}$ inter-ensayo $<10 \%$ para todos los analitos.

Asimismo, se estimó la resistencia a la insulina mediante el índice HOMA, el cual se calcula a partir de la glucosa en ayuno y los niveles de insulina basal por medio de la siguiente fórmula: RI: glucosa $(\mathrm{mg} / \mathrm{dL})$ * insulina $(\mu \mathrm{U} /$ $m l) / 22,5^{16}$. Además, se evaluó la proporción de niños con SM en relación con la RI de acuerdo a cada uno de los criterios diagnósticos. Lo anterior, con la finalidad de evaluar su empleo como Gold Standard (GS) frente a los criterios diagnósticos de SM evaluados.

Presión arterial: Para la toma de la presión arterial, se utilizó la media de dos mediciones realizadas con esfigmomanómetros de mercurio previamente calibrados $^{17}$. La medición de la presión arterial sistólica y diastólica ( $\mathrm{mmHg}$ ) se llevó a cabo mediante esfigmomanómetros de mercurio previamente calibrados y personal capacitado, utilizando brazaletes estándar (ALPK2, Tokio, Japón). La toma de presión sistólica se basó en el primer ruido de Korotkoff, mientras que la de presión diastólica en el quinto ruido. Se llevaron a cabo dos mediciones (con al menos dos minutos de diferencia) para obtener un promedio, para esto, el sujeto debió permanecer sentado en reposo por un mínimo de cinco minutos previos a la toma de la medición según las pautas publicadas en las Guías USA 2004. Finalmente se realizó el registro con una precisión de $2 \mathrm{mmHg}$.

Actividad física y hábitos dietéticos: Para evaluar la actividad física, se utilizó un cuestionario adaptado y versión actualizada de un cuestionario previamente validado, de una población de ingresos bajos y medios de la Ciudad de México, con valores de reproducibilidad de $\mathrm{r}=0,38$ a actividad física moderada y $\mathrm{r}=0,55$ a actividad física vigorosa ${ }^{18}$. Este cuestionario recopiló información sobre los periodos de actividad e inactividad física (horas/d) durante el mes previo a la entrevista, con los cuales se obtuvieron los equivalentes metabólicos de actividad física por hora a la semana (Mets/hora/ semana). En relación a los hábitos dietéticos, se utilizó un cuestionario de frecuencia de consumo de alimentos semi cuantitativo que recopiló información acerca del consumo de 107 alimentos durante el mes previo a la entrevista. A partir de la frecuencia reportada y la cantidad de alimento indicada, se calcularon los gramos al día de consumo de los alimentos, con lo cual se estimó la ingesta de energía dietética y macronutrimentos.

\section{Criterios Diagnósticos de Síndrome Metabólico}

La clasificación de SM se realizó con los puntos de corte de cada uno de los criterios diagnósticos, considerando a un niño con SM en caso de presentar tres o más de los cinco componentes en los criterios de NCEP - ATP III, Cook, et al; Ford, et al; Duncan, et al; Ferranti, et al; y Cruz, et al..$^{1,6,12-16}$ y dos o más componentes con la presencia de obesidad central en el criterio de IDF.

El GS se conformó combinando los cinco criterios diagnósticos (NCEP-ATPIII; Cook, et al; Ford, et al y Duncan, et al; Ferranti, et al; Cruz, et al; e IDF) $)^{1,6,12-16}$. La categoría de riesgo de SM del GS se realizó agrupando la población en riesgo determinada por cada uno de los criterios diagnósticos.

Para determinar la nueva propuesta del criterio diagnóstico de SM en niños mexicanos, se analizaron aquellos componentes incluidos en los criterios 
diagnósticos evaluados, tomando en cuenta CC, PA (sistólica y diastólica) y parámetros bioquímicos (TGC,c-HDL,GA) con los distintos puntos de corte ${ }^{16,19}$. Asimismo, se evaluaron componentes no incluidos en los criterios diagnósticos como AF, IN, e índice HOMA empleando puntos de corte mencionados por la literatura ${ }^{10,20-22}$. Otros componentes evaluados dentro de la nueva propuesta del criterio diagnóstico fueron el $\mathrm{ICC}^{23-25}, \mathrm{IMC}^{26,27}$, presión arterial media $(\mathrm{PAM})^{28}, \mathrm{CT}^{22}$, y C-LDL ${ }^{22}$.
Se realizaron 348 combinaciones con los componentes mencionados previamente tomando en consideración los distintos puntos de corte de cada uno y evaluando el diagnóstico de SM al presentar dos o más, tres o más, o cuatro o más de los cinco, seis o siete componentes considerados para el diagnóstico de SM en niños; y para cada combinación se analizaron los valores de pruebas de sensibilidad diagnóstica (Tabla 1). Se realizaron combinaciones de nuevas propuestas de criterios diagnósticos categorizándolas de la siguiente forma:

Tabla 1. Caracterización de cada uno de los criterios diagnósticos de síndrome metabólico en niños y adolescentes.

\begin{tabular}{|c|c|c|c|c|c|}
\hline \multicolumn{6}{|c|}{ CATEGORIZACIÓN } \\
\hline Variable & $\begin{array}{l}\text { Cook et al., } \\
\text { Ford et al., } \\
\text { Duncan et al. }\end{array}$ & NCEP - ATP III. & Ferranti et al. & Cruz et al. & IDF. \\
\hline $\mathrm{CC}^{\mathrm{a}}$ & $\begin{array}{c}\geq \mathrm{p} 90(82,8 \mathrm{~cm}) \\
\text { en riesgo* }\end{array}$ & $*$ & $\begin{array}{l}>\text { p75 }(74,4 \mathrm{~cm}) \\
\text { en riesgo }\end{array}$ & $*$ & $*$ \\
\hline $\mathbf{T G C}^{\mathbf{b}}$ & $\begin{array}{l}\geqq 110 \mathrm{mg} / \mathrm{dL} \\
\text { en riesgo* }\end{array}$ & $*$ & $\begin{array}{l}\geqq 100 \mathrm{mg} / \mathrm{dL} \\
\text { en riesgo }\end{array}$ & $\begin{array}{c}\geqq p 90(154 \mathrm{mg} / \mathrm{dL}) \\
\text { en riesgo }\end{array}$ & $\begin{array}{l}\geqq 150 \mathrm{mg} / \mathrm{dL} \\
\text { en riesgo }\end{array}$ \\
\hline C-HDL ${ }^{c}$ & $\begin{array}{l}\leqq 40 \mathrm{mg} / \mathrm{Dl} \\
\text { en riesgo* }\end{array}$ & $*$ & $\begin{array}{c}<50 \mathrm{mg} / \mathrm{dL} \\
\text { en riesgo }\end{array}$ & $\begin{array}{c}\leqq \mathrm{p} 10(35 \mathrm{mg} / \mathrm{dL}) \\
\text { en riesgo }\end{array}$ & $\begin{array}{c}<40 \mathrm{mg} / \mathrm{dL} \\
\text { en riesgo }\end{array}$ \\
\hline $\mathbf{P A}^{\mathbf{d}}$ & $\begin{array}{l}\text { Sist/Diast } \\
\geqq \mathrm{p} 90(110 / 79 \mathrm{mmHg}) \\
\text { en riesgo* }\end{array}$ & $*$ & $\begin{array}{c}\text { Sist/Diast } \\
>\text { p90 }(111 / 80 \mathrm{mmHg}) \\
\text { en riesgo }+\end{array}$ & + & $\begin{array}{c}\text { Sist } / \text { Diast } \\
\geqq 130 / 85 \mathrm{mmHg} \text { en } \\
\text { riesgo }\end{array}$ \\
\hline $\mathbf{G A}^{\mathrm{e}}$ & $\begin{array}{l}\geqq 110 \mathrm{mg} / \mathrm{dL} \\
\text { en riesgo* }\end{array}$ & $\begin{array}{l}\geqq 100 \mathrm{mg} / \mathrm{dL} \\
\text { en riesgo } \pm\end{array}$ & $*$ & \pm & \pm \\
\hline
\end{tabular}

${ }^{\mathrm{a}}$ circunferencia de cintura, ${ }^{\mathrm{b}}$ Triglicéridos, ${ }^{\mathrm{c}}$ Lipoproteína de alta densidad, ${ }^{\mathrm{d}}$ Presión arterial, ${ }^{\mathrm{e}} \mathrm{Glucosa}$ en ayuno, ${ }^{\mathrm{e}}$ Sístolica/ diastólica, percentil, *: Misma categorización de Cook et al, Ford et al, Duncan et al, +: Misma categorización que Ferrati et al, \pm : Misma categorización que NCEP-ATPIII.

- Criterios diagnósticos sin necesidad de parámetros bioquímicos: mediciones antropométricas, PA (sistólica y diastólica), y PAM.

- Criterios diagnósticos eligiendo únicamente un parámetro bioquímico: mediciones antropométricas, PA (sistólica y diastólica), PAM, y GA.

- Criterios diagnósticos eligiendo dos parámetros bioquímicos: mediciones antropométricas, PA (sistólica y diastólica), PAM, GA, TGC, CT, C-LDL, y C-HDL.

\section{Análisis estadístico}

Se calculó un poder estadístico del 100\% considerando un nivel de significancia de 0.05 y una prevalencia de SM del $20 \%$, la cual fue reportada para niños y adolescentes mexicanos de acuerdo a información previa del 2009 mostrada por Wacher-Rodarte, et al. ${ }^{20}$, utilizando el criterio diagnóstico de Ferranti, et al. ${ }^{14}$ El análisis estadístico descriptivo estuvo compuesto por: a). Descripción de las características sociodemográficas 129 de los niños; b). Media y desviación estándar de los componentes del perfil metabólico (GA, CT, C-LDL, C-HDL, TGC, IN, presión arterial sistólica y diastólica, circunferencia de cintura, e IMC); y c). Proporción de sujetos con síndrome metabólico para cada uno de los criterios diagnósticos. Las variables cuantitativas continuas fueron categorizadas en terciles, cuartiles y/o tomando como referencia un punto de corte establecido en la literatura.

Se calculó la sensibilidad, especificidad, valores predictivos positivo (VPP) y negativo (VPN) y razón de verosimilitudes para el diagnóstico clínico de síndrome metabólico de cada uno de los criterios diagnósticos. El mejor punto de corte de cada uno de los componentes de los criterios diagnósticos se determinó mediante la combinación de sensibilidad, especificidad y la mayor área bajo la curva de características operativas para el receptor (ROC). Todas las pruebas estadísticas se realizaron con un nivel de significancia de 0,05 . Además, se realizó un análisis de regresión logística en donde se compararon los VPP y VPN de los diferentes 
criterios diagnósticos y se determinaron los coeficientes de los componentes de los criterios diagnósticos, con el fin de identificar aquellos con mejor valor predictivo en nuestra población de estudio.

Con relación al GS, se calculó la sensibilidad, especificidad, VPP, VPN, y razón de verosimilitudes comparando al GS frente a cada uno de los criterios diagnósticos. Se analizó la conformación del GS incluyendo al criterio diagnóstico identificado con mayor sensibilidad y especificidad (de Ferranti, et a ${ }^{14}$.) frente al resto de los criterios diagnósticos evaluados y sin incluirlo dentro de la conformación del GS, para descartar que fuera este quien estuviera determinando el poder que tenía el GS. También se calculó la sensibilidad, especificidad, VPP y VPN, y razón de verosimilitudes para comparar al GS frente a cada una de las 348 propuestas de criterios diagnósticos de $\mathrm{SM}$. Se evaluó la relación costo-beneficio de cada una de las propuestas elegidas determinando el costo que implicaría utilizar dicho criterio para el diagnóstico de SM. El costo de cada propuesta se determinó sumando el costo individual las pruebas bioquímicas que conformaban cada uno de los criterios diagnósticos propuestos. Se eligieron aquellas propuestas de criterios diagnósticos que pudieran estar cercanos a la sensibilidad y especificidad frente al GS y que pudieran definirse de mejor costo-beneficio para el diagnóstico de SM en niños mexicanos.

\section{Resultados}

De los 2599 niños, $53,0 \%$ eran mujeres y $47,0 \%$ hombres, con una edad entre 6 y 16 años con media de $9,4 \pm 2.3$ años, con un IMC promedio de $19,6 \pm 4.2 \mathrm{~kg} / \mathrm{m} 2$ y CC de $66,6 \pm 11.9 \mathrm{~cm}$; la media de PA sistólica fue de $98,6 \pm 11.2 \mathrm{mmHg}$ y diastólica de $66,2 \pm 8,9 \mathrm{mmHg}$. Con respecto a los parámetros bioquímicos la población presentó valores de TG, C-LDL, y GA dentro de los rangos considerados normales en la población pediátrica $^{10,17,18}$ con excepción del CT, c-HDL e IN (Tabla 2).

Tabla 2. Características generales de niños y adolescentes de la Cuidad de México.

\begin{tabular}{|c|c|c|c|c|c|c|}
\hline Variable & $\mathbf{n}$ & $\%$ & Media & DE. & Min. & Max. \\
\hline \multicolumn{7}{|l|}{ Edad (años): } \\
\hline Hombre & 1223 & 47.0 & 9.3 & 2.2 & 6 & 16 \\
\hline Mujer & 1376 & 52.9 & 9.56 & 2.33 & 6 & 16 \\
\hline $\operatorname{IMC}^{\mathrm{a}}\left(\mathrm{kg} / \mathrm{m}^{2}\right)$ & 2596 & 99.9 & 19.58 & 4.25 & 9.23 & 40.92 \\
\hline Circunferencia cintura $(\mathrm{cm})$ & 2595 & 99.8 & 66.34 & 11.97 & 43.8 & 115.2 \\
\hline Colesterol total $(\mathrm{mg} / \mathrm{dL})$ & 2474 & 95.2 & 157.08 & 50.41 & 54 & 437 \\
\hline Triglicéridos (mg/dL) & 2474 & 95.2 & 92.48 & 47.82 & 10 & 444 \\
\hline Colesterol LDL ${ }^{\mathrm{b}}(\mathrm{mg} / \mathrm{dL})$ & 2474 & 95.2 & 101.81 & 26.90 & 13 & 241 \\
\hline Colesterol HDL ${ }^{\mathrm{c}}(\mathrm{mg} / \mathrm{dL})$ & 2474 & 95.2 & 50.74 & 12.88 & 16 & 109 \\
\hline \multicolumn{7}{|l|}{ Presión $\operatorname{arterial}^{\mathrm{d}}(\mathrm{mmHg})$ : } \\
\hline Sistólica & 2589 & 99.6 & 98.61 & 11.16 & 60 & 160 \\
\hline Diastólica & 2588 & 99.6 & 66.17 & 8.93 & 30 & 105 \\
\hline Glucosa en ayuno $(\mathrm{mg} / \mathrm{dL})$ & 2474 & 95.2 & 81.86 & 9.29 & 45 & 117 \\
\hline Insulina $(\mu \sigma / \mathrm{mL})$ & 1407 & 54.1 & 7.59 & 7.64 & 0.2 & 105.64 \\
\hline Actividades en tiempo libre (mets $\mathrm{min} / \mathrm{sem}$ ) & 2478 & 95.3 & $2,867.9$ & $2,624.2$ & 0 & $24,428.5$ \\
\hline Actividad física al aire libre ( $\mathrm{min} / \mathrm{sem})$ & 2478 & 95.3 & 441.6 & 403.05 & 0 & 2175 \\
\hline \multicolumn{7}{|l|}{ Consumo de Energía: } \\
\hline Total (kilocalorías) & 2522 & 97 & $3,186.0$ & $1,030.6$ & 623.8 & $6,386.0$ \\
\hline Carbohidratos (\%) & 2522 & 97 & 57.20 & 5.07 & 34.3 & 76.0 \\
\hline Lípidos (\%) & 2522 & 97 & 28.28 & 4.60 & 11.4 & 53.7 \\
\hline Proteínas $(\%)$ & 2522 & 97 & 14.52 & 1.87 & 6.2 & 24.5 \\
\hline
\end{tabular}

ándice de masa corporal, blipoproteína de baja densidad, 'Lipoproteína de alta densidad, dpresión arterial.

Al evaluar los criterios diagnósticos de SM, con sus respectivos puntos de corte de cada uno de los componentes, se mostraron diferencias estadísticamente significativas de los criterios diagnósticos entre sí $(\mathrm{p}<0,05)$. De acuerdo a la relación porcentual de CC se apreció una proporción del $10.0 \%$ de individuos en riesgo de $\mathrm{SM}$ (CC $\geq$ percentil 90) (de acuerdo a los criterios de Cook, et al., Ford, et al; Duncan, et al; Cruz, 
et al; NCEP - ATP III; e IDF $\left.{ }^{1,6,12-16}\right)$, en comparación con $24,6 \%$ de individuos en riesgo de SM con el criterio de Ferranti, et al $(\mathrm{p}=0.0001)$.

Se observaron concentraciones de $\mathrm{TG}$ con riesgo de SM en una proporción de individuos del $24,2 \%$ con TG $\geq 110 \mathrm{mg} / \mathrm{dL}$ en comparación con el criterio de Ferranti, et $\mathrm{al}^{14}$ con $30,3 \%$ individuos en riesgo $(\mathrm{TG} \geq 100 \mathrm{mg} / \mathrm{dL})$, 9,6\% de los individuos con el criterio de Cruz, et al. ${ }^{15}$, y $10,4 \%$ de los individuos según el criterio diagnóstico de $\operatorname{IDF}^{6}(\mathrm{p}=0,0001)$.

Esto es consistente con respecto a los criterios diagnósticos de SM de Cook, et al., Ford, et al., Duncan, et al; y NCEP - ATP III ${ }^{1,6,12-16}$ considerando la relación porcentual de TG con el Riesgo de SM. Además se observó que una concentración de c-HDL $\leq 40 \mathrm{mg} / \mathrm{dL}$, se encontraba en un $20,9 \%$ de los individuos los cuales se clasificaron con riesgo de presentar SM (c-HDL) en comparación con 46,3\% individuos con el criterio de Ferranti, et al. ${ }^{14}$ (c-HDL <50mg/dL), 10,9\% de los individuos según el criterio de Cruz, et al. ${ }^{15}$, y 18,4\% de los individuos con el criterio de $\operatorname{IDF}^{6}(\mathrm{p}=0,0001)$.

Al evaluar de PA sistólica, se observó una mayor proporción de individuos $(20,3 \%)$ con PA sistólica $\geq$ percentil $90(110 \mathrm{mmHg})$ en comparación con $9,7 \%$ de los individuos con los criterios de Ferranti, et al. ${ }^{14} \mathrm{y}$ Cruz, et al. ${ }^{15}$, y $1 \%$ de los individuos según el criterio de $\operatorname{IDF}^{6}(p=0,0001)$.

Con respecto a las concentraciones de glucosa, se observó una relación del $0,4 \%$ de los individuos con una glucosa $\geq 110 \mathrm{mg} / \mathrm{dL}$ en comparación con $2,5 \%$ individuos según los criterios de Cruz, et al. ${ }^{15}$ NCEP - ATP III, e IDF 6 con una glucosa $\geq 100 \mathrm{mg} / \mathrm{dL}$ $(p=0,0001)$. Por lo que al determinar la proporción de niños diagnosticados con SM de acuerdo a cada uno de los criterios diagnósticos, se observó una mayor proporción de individuos diagnosticados con el criterio de Ferranti, et al. ${ }^{14}(12,9 \%)$ (Tabla 3).

Tabla 3. Distribución de los niños y adolescentes de acuerdo a cada uno de los criterios diagnósticos de síndrome metabólico evaluados.

\begin{tabular}{|c|c|c|c|c|c|c|c|}
\hline \multirow{2}{*}{ Criterio diagnóstico } & \multicolumn{2}{|c|}{$\mathbf{S M}^{\mathbf{a}}$} & \multicolumn{2}{|c|}{ Sin SM } & \multirow[t]{2}{*}{ Valor $p$} & \multicolumn{2}{|c|}{ Total } \\
\hline & $\mathbf{n}$ & $\%$ & $\mathbf{n}$ & $\%$ & & $\mathbf{n}$ & $\%$ \\
\hline Ferranti et al & 335 & 12,9 & 2264 & 87,1 & 0,0001 & 2599 & 100 \\
\hline NCEP - ATPIII & 133 & 5,1 & 2466 & 94,9 & 0,0001 & 2599 & 100 \\
\hline Cook et al., Ford et al., Duncan et al & 124 & 4,8 & 2475 & 95,2 & 0,0001 & 2599 & 100 \\
\hline Cruz et al. & 67 & 2,6 & 2532 & 97,4 & 0,0001 & 2599 & 100 \\
\hline IDF. & 53 & 2,0 & 2546 & 97,9 & 0,0001 & 2599 & 100 \\
\hline IDF (10 a 15 años). & 40 & 3,3 & 1166 & 96,7 & 0,0001 & 1206 & 100 \\
\hline IDF $(\geq 16$ años $)$ & 0 & 0,0 & 6 & 100 & 0,0001 & 6 & 100 \\
\hline
\end{tabular}

${ }^{a}$ Síndrome Metabólico.

Se calculó sensibilidad, especificidad, VPP y VPN, y razón de verosimilitudes comparando al índice HOMA como GS frente a cada uno de los criterios diagnósticos evaluados la proporción de enfermos con resultado positivo obtenida en todos los criterios diagnósticos fue muy baja; teniendo un $0,03 \%$ con el criterio de $\operatorname{IDF}^{6}(p=0,285)$, hasta 0,15 con el criterio de Ferranti, et al. ${ }^{14}(\mathrm{p}=0,206)$. En cuanto a la proporción de sanos con resultado negativo, la mayoría de los criterios diagnósticos presentaron valores elevados, obteniéndose proporciones de 0.88 con el criterio de Ferranti, et al. ${ }^{14}$ hasta 0,99 con los criterios de Cruz, et al. ${ }^{15}$ e $\mathrm{IDF}^{6}$.

Por otra parte, se estimó la sensibilidad, especificidad, VPP y VPN, y razón de verosimilitudes comparando al GS conformado por los cinco criterios diagnósticos evaluados, frente a cada uno de ellos (Tabla 4). La proporción de enfermos con resultado positivo obtenida en todos los criterios diagnósticos fue mayor que la obtenida con el índice HOMA; se obtuvieron proporciones de 0,16 con el criterio de $\mathrm{IDF}^{6}$ $(\mathrm{p}=0,0001)$, hasta 0,98 con el criterio de Ferranti, et $\mathrm{al}^{14}$. $(\mathrm{p}=0,0001)$. En cuanto a la proporción de sanos con resultado negativo, todos los criterios diagnósticos presentaron una proporción de 1,00. El criterio diagnóstico de Ferranti, et al. ${ }^{14}$ fue identificado como el criterio con mayor sensibilidad $(0,98)$, especificidad $(1,00)$, VPP $(1,00)$, y VPN $(1,00)$ frente al GS, en comparación con el resto de los criterios diagnósticos evaluados. 
Estudio de validación de cuatro diferentes criterios para el diagnóstico de síndrome metabólico en población infantil

Tabla 4. Sensibilidad, especificidad, valores predictivos y razón de verosimilitudes de cada criterio diagnóstico de síndrome metabólico frente al estándar de oro (GS)

\begin{tabular}{lcccccc}
\hline & & Sensibilidad & Especificidad & VPP $^{\mathbf{a}}$ & VPN $^{\mathbf{b}}$ & $\boldsymbol{p}$ \\
\hline Ferranti et al* & GS & $\mathbf{0 , 9 8}$ & $\mathbf{1 , 0 0}$ & 1,00 & 1,00 & 0,0001 \\
& GS sin Ferranti* & $\mathbf{0 , 9 5}$ & $\mathbf{0 , 9 2}$ & 0,38 & 1,00 & 0,0001 \\
NCEP-ATPIII & GS & 0,39 & $\mathbf{1 , 0 0}$ & 1,00 & 0,92 & 0,0001 \\
& GS sin Ferranti* & $\mathbf{1 , 0 0}$ & $\mathbf{1 , 0 0}$ & 1,00 & 1,00 & 0,0001 \\
Cook, Ford, Duncan et al & GS & 0,36 & $\mathbf{1 , 0 0}$ & 1,00 & 0,91 & 0,0001 \\
& GS sin Ferranti* & 0,93 & $\mathbf{1 , 0 0}$ & 1,00 & 1,00 & 0,0001 \\
Cruz et al. & GS & 0,20 & $\mathbf{1 , 0 0}$ & 1,00 & 0,89 & 0,0001 \\
& GS sin Ferranti* & 0,50 & $\mathbf{1 , 0 0}$ & 1,00 & 0,97 & 0,0001 \\
IDF. & GS & 0,16 & $\mathbf{1 , 0 0}$ & 1,00 & 0,89 & 0,0001 \\
& GS sin Ferranti* & 0,40 & $\mathbf{1 , 0 0}$ & 1,00 & 0,97 & 0,0001 \\
IDF (10 a 15años) & GS & 0,20 & $\mathbf{1 , 0 0}$ & 1,00 & 0,89 & 0,0001 \\
& GS sin Ferranti* & 0,38 & $\mathbf{1 , 0 0}$ & 1,00 & 0,95 & 0,0001 \\
\hline
\end{tabular}

${ }^{\mathrm{a}}$ Valor predictivo positivo, ${ }^{\mathrm{b}}$ Valor predictivo negativo

Al realizar nuevamente el análisis eliminando del GS al criterio diagnóstico de Ferranti et al. ${ }^{14}$, las proporciones de sensibilidad y especificidad siguieron siendo altas, con proporciones de 0,95 y 0,92 respectivamente.

Además, se calculó la sensibilidad, especificidad, VPP y VPN, y razón de verosimilitudes comparando al GS y al criterio diagnóstico de Ferranti, et al. ${ }^{14}$, confrontado a cada una de las 348 propuestas de criterios diagnósticos de SM. Las propuestas elegidas, con alta sensibilidad y especificidad, se conformaron por el diagnóstico de SM al presentar tres o más de los siguientes componentes constituidos por tres diferentes mediciones antropométricas (CC, ICC e IMC), PA sistólica y/o PA diastólica y/o PAM, y dos distintas mediciones de parámetros bioquímicos (GA y TGC); dichas propuestas presentaron una sensibilidad de 0,84 a 0,89 , y una especificidad de 0,90 a 0,93 . Las tres propuestas elegidas con mayor sensibilidad y especificidad, frente a los demás criterios diagnósticos propuestos, fueron los siguientes:

- Propuesta 3: $\quad$ CC $>$ percentil75, PA diastólica $>$ percentil $90, \mathrm{TGC} \geq 100 \mathrm{mg} / \mathrm{dL}, \mathrm{c}-\mathrm{HDL}$ $\leq 40 \mathrm{mg} / \mathrm{dL}$, índice HOMA $\geq 2,5$ : con Sensibilidad de 0,89 y Especificidad de 0,93.

- Propuesta 159: CC $>$ percentil 75, ICC $>0,95$ en hombres y $>0,85$ en mujeres, IMC $\geq$ percentil 85 , PA sistólica $\geq$ percentil 90, PAM $\geq$ percentil 90 , GA $\geq 100 \mathrm{mg} / \mathrm{dL}, \mathrm{TGC} \geq 100 \mathrm{mg} / \mathrm{dL}$ : con Sensibilidad de 0,89 y Especificidad de 0,90.

- Propuesta 160: CC $>$ percentil 75, ICC $>0,95$ en hombres y $>0,85$ en mujeres, $\mathrm{IMC} \geq$ percentil 85 ,
PA sistólica $\geq$ percentil 90, PA diastólica $\geq$ percentil $90, \quad$ GA $\geq 100 \mathrm{mg} / \mathrm{dL}, \quad \mathrm{TGC} \geq 100 \mathrm{mg} / \mathrm{dL}$ : con Sensibilidad de 0,89 y Especificidad de 0,90.

$\mathrm{Al}$ evaluar la relación costo-beneficio de cada una de las propuestas elegidas, se determinó el costo que implicaría utilizar dicho criterio para el diagnóstico de SM. El análisis anterior se realizó investigando costos de tiras reactivas de la marca "Accutrend Plus" y "CardioCheck P.A." para la determinación de parámetros bioquímicos de GA, CT, C-LDL, C-HDL, y TGC. Al determinar el costo del criterio diagnóstico de Ferranti, et al. ${ }^{14}$ se obtuvo un costo total de $\$ 84,45$ (EUR $\$ 4,83)$ en comparación con MX\$498,45 (EUR \$28,51) con la propuesta 3, y MX\$35,85 (EUR $\$ 2,05)$ con las propuestas 159 y 160 (Tabla 5).

Se eligió aquella propuesta de criterio diagnóstico cercana a la sensibilidad y especificidad del GS y que pudiera definirse de mejor costo-beneficio para el diagnóstico de SM en niños mexicanos. La propuesta ad hoc para el diagnóstico de síndrome metabólico en niños mexicanos en la que se alcanzó una alta sensibilidad y especificidad, y un bajo costo en comparación con las demás propuestas fue:

- Propuesta 160: $\mathrm{CC}>$ percentil $75, \mathrm{ICC}>0.95$ en hombres y $>0.85$ en mujeres, IMC $\geq$ percentil 85 , PA sistólica $\geq$ percentil 90 , PA diastólica $\geq$ percentil $90, \mathrm{GA} \geq 100 \mathrm{mg} / \mathrm{dL}, \mathrm{TGC} \geq 100 \mathrm{mg} / \mathrm{dL}$ :

- Sensibilidad de 0,89 y Especificidad de 0,90.

- Costo de $\$ 35,85$ pesos M.N por individuo. 
Tabla 5. Costo de propuestas elegidas por su alta sensibilidad y especificidad.

\begin{tabular}{|c|c|c|c|c|}
\hline & \multicolumn{4}{|c|}{ PROPUESTA } \\
\hline Componentes & Estándar de oro & Ferranti et al. & Criterio 3 & Criterio 160 \\
\hline Cintura (cms) & $>\mathrm{p}^{\mathrm{i}} 75=74,4>\mathrm{p} 90=82,8$ & $>\mathrm{p} 75=74,4$ & $>\mathrm{p} 75=4,4$ & $>$ p75 $=74,4$ \\
\hline PAS $^{\text {a }}(\mathbf{m m H g})$ & $>\mathrm{p} 90=111, \geq \mathrm{p} 90=110, \mathrm{o}>130$ & $>\mathrm{p} 90=111$ & -------- & $\geq p 90=110$ \\
\hline $\mathbf{P A D}^{\mathrm{b}}(\mathbf{m m H g})$ & $>\mathrm{p} 90=80, \geq \mathrm{p} 90=79, \mathrm{o}>85$ & $>\mathrm{p} 90=80$ & $>\mathrm{p} 90=80$ & $\geq p 90=79$ \\
\hline $\mathbf{G A}^{\mathrm{c}}(\mathrm{mg} / \mathrm{dL})$ & $\geq 110, \mathrm{o} \geq 100$ & $\geq 110$ & ------- & $\geq 100$ \\
\hline C-HDL ${ }^{d}(\mathrm{mg} / \mathrm{dL})$ & $<50,<40$ o $<$ p $10=35$ & $<50$ & $\leq 40$ & ------- \\
\hline $\operatorname{TGC}^{\mathrm{e}}(\mathrm{mg} / \mathrm{dL})$ & $\geq 100,>110,>\mathrm{p} 90=154, \mathrm{o}>150$ & $\geq 110$ & $\geq 100$ & $\geq 100$ \\
\hline HOMA $^{\mathrm{f}}$ & ------- & ------- & $\geq 2,5$ & ------- \\
\hline $\mathrm{IMC}^{\mathrm{g}}$ & -------- & -------- & -------- & $\geq \mathbf{p 8 5}$ \\
\hline $\mathrm{ICC}^{\mathrm{h}}$ & ------- & -------- & -------- & $\mathbf{H}>\mathbf{0 , 9 5} \quad \mathrm{M}>\mathbf{0 , 8 5}$ \\
\hline costo & $\$ 84,45$ & $\$ 84,45$ & $\$ 498,5$ & $\$ 35,85$ \\
\hline Sensibilidad & 1 & 0,98 & 0,89 & 0,89 \\
\hline Especificidad & 1 & 1 & 0,93 & 0,9 \\
\hline
\end{tabular}

aPresión arterial sistólica, ${ }^{\mathrm{b}}$ Presión arterial diastólica, ${ }^{\mathrm{c}} \mathrm{Glucosa}$ en ayunas, ${ }^{\mathrm{d}}$ Lipoproteína de alta densidad, ${ }^{\mathrm{e}}$ Triglicéridos, ${ }^{\mathrm{f}}$ Indice de resistencia a la insulina, Indice de masa corporal, hIndice de cadera-cintura.

\section{Discusión}

Existen diferencias en los criterios diagnósticos de SM en niños y adolescentes que varían según los autores y los comités de expertos ${ }^{29}$. Las divergencias se enfocan en los componentes que deben o no incluirse, así como en los puntos de corte adecuados para su definición. Las diferencias expuestas con respecto a la diversidad de definiciones diagnósticas de SM en niños y adolescentes y la determinación de la prevalencia de SM en la población, pudo observarse en estudios como el de Reinehr T, et al. ${ }^{30} \mathrm{El} \mathrm{SM}$ se definió de acuerdo a criterios propuestos para niños y adolescentes (Cook, Ferranti, Viner, Weiss ${ }^{1,6,12-16}$ ) y de acuerdo a criterios para adultos (ATPIII, EGIR, IDF, OMS ${ }^{1,6,12-16}$ ). Se reportó una variación significativa $(p<0,001)$ en la prevalencia de SM entre las diferentes definiciones, encontrándose una prevalencia desde 6\% (OMS) al 39\% (De Ferranti,et al. $\left.{ }^{14}\right)$. Cabe señalar que en las definiciones de Cook y Ferranti ${ }^{12,14}$ se detectó mayor frecuencia de SM (resultado similar a nuestro análisis reportado) debido a los puntos de corte empleados. Sólo 9\% de los niños cumplieron con los cuatro criterios de SM para niños y adolescentes, mientras que tan sólo el 2\% cumplió con los ocho criterios estudiados de SM. Sin embargo, este estudio encontró una asociación de la resistencia a la insulina y el grado de obesidad con la ocurrencia de SM, independientemente de la definición empleada. Otro estudio realizado por Camarillo-Romero, et al. ${ }^{31}$ determinó diferencias entre las definiciones de NCEPATPIII e IDF en 575 adolescentes mexicanos (de 14 a 16 años) reportando una mayor prevalencia de SM de acuerdo a la definición de NCEP-ATPIII $(18,6 \%)$ en comparación con la definición de IDF $(8,2 \%)$ $(\mathrm{p}<0.001)^{27}$. Así mismo en un estudio realizado en escolares colombianos observaron que la prevalencia de SM fue de $0.3 \%, 6.3 \%, 7.8 \%$, y $11.0 \%$ dependiendo de los criterios utilizados IDF, Cook, et al., Ford, et al., y de Ferranti, et al., respectivamente ${ }^{32}$. Estos reportes sustentan la importancia del estudio realizado para validar los criterios y establecer uniformidad para el diagnóstico de SM en población infantil.

En el presente estudio se mostraron dichas divergencias al encontrar una mayor proporción de individuos diagnosticados con el criterio de Ferranti, et al. ${ }^{14}(12,9 \%)$ en comparación con $4,8 \%$ de Cook, et al., Ford, et al. y Duncan, et al. ${ }^{12-14} ; 2,6 \%$ de Cruz, et al. ${ }^{15}$; $5,1 \%$ de NCEP-ATPIII, y $2 \%$ de $^{2}$ IDF $^{6}(\mathrm{p}<0,001)$. Las diferencias entre las proporciones de SM reportadas se deben principalmente a los distintos puntos de corte para evaluar la composición corporal por medio de CC o IMC empleando tanto percentiles como puntajes z; valores de dislipidemia con diferentes criterios para TGC y C-HDL empleando puntos de corte en percentiles o mg/dL; y distintos puntos de corte para definir HTA.

El tamaño de muestra utilizado en este estudio permitió conseguir un poder estadístico suficiente para garantizar la estabilidad de los resultados, asegurando que estos no se deban a efectos del azar, pudiendo prever factibilidad en las diferencias observadas entre 
las diferentes definiciones diagnósticas de SM y cada uno de los componentes evaluados. Las mediciones antropométricas se realizaron en toda la población y no fueron establecidas en base a un autoreporte de los participantes, evitando así los posibles sesgos de percepción física y de recuerdo. Asimismo, todas las mediciones se realizaron por personal capacitado $\mathrm{y}$ estandarizado, empleando técnicas e instrumentos previamente validados.

El presente estudio permitió evaluar diferentes definiciones (NCEP-ATPIII; Cook, et al. Ford, et al. y Duncan, et al; Ferranti et al; Cruz et al; e IDF ${ }^{1,6,12-16}$ ) para el diagnóstico de síndrome metabólico, identificando aquellos componentes con mejor valor predictivo. De esta forma, se propone un criterio ad hoc para el diagnóstico de SM en la población de niños mexicanos, el cual presenta una alta sensibilidad $(0,89)$ y especificidad $(0,90)$ frente al GS aplicado. Dicho criterio diagnóstico propuesto, contiene una elección de componentes sencillos y de bajo costo (medición de circunferencia de cintura, ICC, IMC, presión arterial sistólica y diastólica, glucosa en ayuno, y triglicéridos) que facilitará su aplicación en las instituciones de salud. Lo anterior permitirá identificar apropiadamente el riesgo futuro de enfermedades cardiovasculares, así como unificar y homogeneizar el diagnóstico, tratamiento y pronóstico poblacional que permitirá reducir los índices de morbimortalidad en la población mexicana. Se sugiere la realización de mayores estudios en el futuro que aborden aspectos referentes al diagnóstico certero y unificado de síndrome metabólico en niños.

Debido a que no se encontró relación alguna entre las variables de actividad física y consumo de alimentos con el riesgo de presentar SM, dichas variables no se agregaron en el análisis final del estudio.

\section{Agradecimientos}

Los autores agradecen los integrantes de la Unidad de Bioquímica-IMSS por la asistencia técnica en los ensayos Bioquímicos y ELISA; al grupo de Epidemiología genética del INSP quienes se encargaron de la recolección de la información y mediciones antropométricas.

\section{Consideraciones éticas}

El presente estudio fue aprobado por los comités de ética, bioseguridad e investigación del Instituto Nacional de salud Pública (INSP). Fue financiado por el Consejo Nacional de Ciencia y Tecnología
(CONACyT) de México, en su convocatoria del Fondo Sectorial de Investigación en el área de Salud, Proyecto Núm: 2012-180808 y CONACYT SALUD2005-C02-14412; Proyectos Estratégicos IMSS 20043601-0020; Fundación IMSS, A.C

\section{Conflicto de intereses}

No hay conflictos de intereses de índole laboral, investigación, económicos o morales.

\section{Referencias}

1. Burguete-García AI, Valdés-Villalpando YN, Cruz M. Definitions for the diagnosis of metabolic syndrome in children. Gac Med Mex. 2014; 150: 79-87.

2. Robles-Valdés C. Riesgo cardiovascular y síndrome metabólico en niños y adolescentes. Acta Pediatr Mex. 2011; 32: 1-4.

3. Titmuss, AT, Srinivasan S. Metabolic syndrome in children and adolescents: Old concepts in a young population. J Paediatr Child Health. 2016; 52: 928-934.

4. Peña-Espinoza BI, Granados-Silvestre MA, Sánchez-Pozos K, Ortiz-López MG, Menjivar M. Metabolic syndrome in Mexican children: Low effectiveness of diagnostic definitions. Endocrinol, Diabetes Nutr. 2017; 64: 369-376.

5. Rojas-Martínez R, Aguilar-Salinas CA, JiménezCorona A, Gómez-Pérez FJ, Barquera S, LazcanoPonce E. Prevalence of obesity and metabolic syndrome components in Mexican adults without type 2 diabetes or hypertension. Salud Publica Mex. 2012; 54: 7-12.

6. Parikh RM, Mohan V. Changing definitions of metabolic syndrome. Indian J Endocrinol Metab. 2012; 16: 7-12.

7. Lerman Garber I, Aguilar-Salinas CA, GómezPérez FJ, Reza Albarrán A, Hernández Jiménez S, Vázquez Chávez C, et al. El síndrome metabólico. Posición de la Sociedad Mexicana de Nutrición y Endocrinología, sobre la definición, fisiopatología y diagnóstico. Características del síndrome metabólico en México. Rev Endocrin Nutrición. 2004; 12: 109-122.

8. Macías Tomei C. Síndrome metabólico en niños y adolescentes. Arch Venez Puer Ped. 2009; 72: 30-37.

9. Zimmet P, Alberti G, Kaufman F, Tajima N, Silink M, Arslanian S, et al. The metabolic síndrome in children and adolescents. Lancet. 2007; 369: 2059-2061.

10. Pires Rodríguez MC, Nava Arias AV, Lanzilli P. Síndrome metabólico: prevalencia y factores de riesgo en escolares. Arch Venez Puer Ped. 2009; 72: 47-52. 
11. Lee L, Sanders RA. Metabolic Syndrome. Pediatr Rev. 2012; 33: 459-466.

12. Cook S, Weitzman M, Auinger P, Nguyen M, Dietz WH. Prevalence of a metabolic syndrome phenotype in adolescents: findings from the third National Health and Nutrition Examination Survey, 19881994. Arch Pediatr Adolesc Med. 2003;157(8): 821827. DOI: 10.1001/archpedi.157.8.821.

13. Duncan GE, Li SM, Zhou XH. Prevalence and trends of a metabolic syndrome phenotype among US adolescents, 1999-2000. Diabetes Care. 2004; 27(10): 2438-2443.

14. DeFerrantiSD, GauvreauK, LudwigDS, NewfeldEJ, Newburger JW, Rifai N. Prevalence of the metabolic syndrome in american adolescents: findings from the third national health and nutrition examination survey. Circulation. 2004; 110(16): 2494-2497. doi: 10.1161/01.CIR.0000145117.40114.C7.

15. Cruz ML, Weigensberg MJ, Huang TT, Ball G, Shaibi GQ, Goran MI. The metabolic syndrome in overweight Hispanic youth and the role of insulin sensitivity. J Clin Endocrinol Metab. 2004; 89(1): 108-113.

16. Sharma S, Fleming SE. Use of HbA1C testing to diagnose pre-diabetes in high risk African American children: a comparison with fasting glucose and HOMA-IR. Diabetes Metab Syndr. 2012; 6: 157-162.

17. Kuri Morales P, Lara Esqueda A, Raquel Ortiz Solís GR, De la Cabada Tamez E, Castro Alba PJ, Vital Calvo O, García Torres H. Manual de calibración y mantenimiento de esfigmomanómetros. Subsecretaría de Prevención y Promoción de la Salud Centro Nacional de Vigilancia Epidemiológica y Control de Enfermedades. México Distrito Federal. 2007. p. 1-40.

18. Hernández B, Gortmaker SL, Laird NM, Colditz GA, Parra-Cabrera S, Peterson KE. Validez y reproducibilidad de un cuestionario de actividad e inactividad física para escolares de la Ciudad de México. Salud Publica Mex 2000; 42: 315-323.

19. Gidding SS, Dennison BA, Birch LL, Daniels SR, Gillman MW, Lichtenstein AH, et al. Dietary recommendations for children and adolescents: a guide for practitioners. Pediatrics. 2006; 117: 544-559.

20. Wacher-Rodarte N. Epidemiología del síndrome metabólico. Gac Med Méx. 2009; 145: 384-391.

21. American Diabetes Association. Standards of Medical Care in Diabetes 2013. Diabetes Care. 2013; 36: S11-S66.

22. Burrows R. Prevención del sobrepeso en la niñez: la mejor estrategia para la prevención de las
Enfermedades Crónicas no Transmisibles (ECNT) del adulto. Revista Hospital Clínico Universidad de Chile. 2005; 16: 341-350.

23. Durán P, Piazza N, Agnestein C, Bay L, Cotti A, Trifone $\mathrm{L}$, et al. Consenso sobre factores de riesgo de enfermedad cardiovascular en pediatría. Obesidad. Arch.argent.pediatr. 2005; 103: 262-281.

24. Cabrera-Rode E, Bioti Torres Y, Marichal Madrazo S, Parlá Sardiñas J, Arranz Calzado C, Olano Justiniani $\mathrm{R}$, et al. Índice cintura-cadera contra perímetro cintura para el diagnóstico del síndrome metabólico en niños y adolescentes con familiares de primer grado diabéticos tipo 1. Rev Cubana Endocrinol. 2011; 22: 182-195.

25. Magalhäes EI, Sant'Ana LF, Priore SE, Franceschini Sdo C. Waist circumference, waist/ height ratio, and neck circumference as parameters of central obesity assessment in children. Rev Paul Pediatr. 2014; 32: 273-281.

26. González Garrido JA, Ceballos Reyes GM, Méndez Bolaina DE. Obesidad: más que un problema de peso. Rev Divulg Cient Tec Univ Veracruzana. La Ciencia y el Hombre. 2010; 23(2).

27. Silveira LS, Buonani C, Monteiro PA, Mello Antunes BM, Freitas Júnior IF. Metabolic syndrome: criteria for diagnosing in children and adolescents. Endocrinol Metab Synd. 2013; 2: 1-6.

28. Halpern A, Mancini MC, C Magalhaes ME, Fisberg M, Radominski R, Bertolami MC, et al. Metabolic syndrome, dyslipidemia, hypertension and type 2 diabetes in youth: from diagnosis to treatment. Diabetol Metab Syndr. 2010; 2: 55.

29. Costa RF, Santos NS, Goldraich NP, Barski TF, de Andrade KS, Kruels LFM. Metabolic syindrome in obese adolescents: a comparison of three different diagnostic criteria. J Pediatr (Rio J). 2012; 88: 303-309.

30. Reinehr T, de Sousa G, Toschke AM, Andler W. Comparison of metabolic syndrome prevalence using eight different definitions: a critical approach. Arch Dis Child. 2007; 92: 1067-1072.

31. Camarillo-Romero E, Domínguez García MV, Amaya-Chávez A, Huitrón-Bravo G, Majluf-Cruz A. Dificultades en la clasificación del síndrome metabólico. El ejemplo de los adolescentes en México. Salud Publica Mex. 2010; 52: 524-527.

32. Ramírez-VélezR, AnzolaA, Martínez-Torres J, Vivas A, Tordecilla-Sanders A, Prieto-Benavides D, et al. Metabolic syndrome and associated factors in a population-based Sample of Schoolchildren in Colombia: The FUPRECOL Study. Metab Syndr Relat Disord. 2016; 14(9): 455-462. doi: 10.1089/ met.2016.0058. 